\title{
Some Microbiological Indexes of Pig and Sheep Carcasses
}

\author{
Nicolae STARCIUC*, Ruslan ANTOCI \\ State Agrarian University of Moldova \\ *Corresponding author: n.starciuc@uasm.md \\ Bulletin UASVM Veterinary Medicine 73(1) / 2016, \\ Print ISSN 1843-5270; Electronic ISSN 1843-5378 \\ DOI:10.15835/buasvmcn-vm: 10503
}

\begin{abstract}
The study aimed at assessing the presence and diversity of microorganisms on pig and sheep carcasses during trading. As a result of investigations was established that the contamination of the carcasses is frequently more common with external bacterial flora which takes place immediately after the slaughter of animals. Bacteriological examination of samples taken from the surface and deep of pig and sheep carcasses has shown the risk of contamination with bacterial microorganisms which is more abundant on the carcasses of sheep with predominance of microorganisms types such E. coli and Streptococcus.
\end{abstract}

Keywords: carcasses, colonies, housing, insemination, micro-nutrient environment, samples

\section{INTRODUCTION}

Microorganisms in the meat industry have an important role on modifying the organoleptic and nutritional properties of meat, which through chemical composition is a very favorable environment for the growth of microorganisms. To prevent the harmful effect is necessary to know the different microorganisms that are found in meat and various meat products and the conditions in which they grow and cause spoilage. Thus, the microorganisms that act adversely on food, generally making them unsuitable for human consumption are bacteria that cause putrefaction and mycosis which causing fermentation.

Research carried out on various tissues of meat from healthy animals, which are cut with the rules of aseptic (uninfected) showed in most cases the muscle germ instead were found in organs. Hygienic quality of meat depends on a various number of factors as animal dependent factors and factors dependent on the cutting unit (Papadopoulou 2012; Tian Ding et al., 2010). Live animal shows two main pathways of exposure to bacterial contamination of the environment, namely the skin, which can be covered with a big number of impurities; gastrointestinal tract, nasopharyngeal cavity and external uro-genital ways. Tired animals with low organic strength, microbes can enter the body either through mucosal or by skin where in the blood and from there go get the muscles.

Importantly is that the animals for slaughter to be brought under more hygienic conditions. Washing took animals is effective only if there are impurities lighter skin. When washing heavily soiled animals before slaughter are solubilizedimpurities and therisk of contamination of meat is highest, especially during the skinning. In normal conditions, the largest bacterial load is found in animal digestive pathways. One gram fresh faeces of cattle contain approximately 500,000 of bacteria. Bleeding and gutting late adversely affect quality of meat hygiene, producing intense contamination of the meat. Breaking intestines during evisceration of carcasses are an important source of contamination of meat, 
sometimes with salmonella (Artur, 2010; Tian Ding et al., 2010). In this category the most important sources of bacterial contamination of meat can be included metal hooks, workers hands during the selling period, tables, blades knives, etc. The germs from the surface of the carcasses have multiply rapidly and penetrate very quickly muscles deeply. As penetration microbial flora in depth aerobic germs are replaced with the anaerobes.

The level of microbial load of meat is influenced by temperature, humidity and shelf life. The usual microbial flora of meat contamination is represented by: streptococcal microbial form, Escherichia coli, Proteus vulgaris, Bacillus magaterium, Clostridium perfringens,etc. A part of the microbial flora present on the surface of carcasses may come from polluted atmosphere of workrooms and during the storage (Juneja et al., 2009; So Jung Kim and Dong Sun Lee, 2009). The meet which is ready for public consumption must come only from healthy animals, knowing that a number of infectious animal diseases can be transmitted to humans through contact or consumption of meat (Papadopoulou 2012; So Jung Kim and Dong Sun Lee, 2009).

\section{MATERIALS AND METHODS}

The investigations were carried out in the Department of the Epizootology, Faculty of Veterinary Medicine (Microbiology Laboratory) and in the laboratory of veterinary expertise. The samples were collected from hall nr.3 with destination achievement of meat, carcasses and meat products in Central agricultural market of Chisinau. Basic material investigations were the pig and sheep carcasses. The meat samples were collected to study the microorganisms load over different time periods of the selling. Samples of pig and sheep carcasses were randomly collected from the carcasses delivered in hall nr. 3 for sail in the morning, between 5.00 to 6.00 o'clocks a.m. The samples weight was around 100-150g samples were examined in the laboratory using the bacteriological investigations. After the collection, the samples were storage in refrigerator at $+4^{\circ} \mathrm{C}$ until the time of seeding on nutrient media.

Seeding was done on the following nutrient medium: peptone agar, peptone broth, Endo medium, Sabouraud medium, bismuth sulphite agar, which had been prepared in accordance known standards, then autoclaved and kept in the refrigerator until insemination carried out. Differential staining was performed by Gram and Ziehl-Neelsen methods. Subsequently, the plates inoculated samples were visually checked within 12 hours, and the interpretation of the final result were carried out after 48 hours. In particular the emphasis was put on the following aspects: color, number of colonies, their structure (surface, overgrown substrate, fluffy, shiny, etc.).

\section{RESULTS AND DISCUSSION}

The results of laboratory investigations are shown on the images below.

Fig.1. shown the Petri plates with the "peptone agar" which was inseminated from the surface and deep samples of sheep carcasses. After 48 hours of incubation in thermostat at $+37{ }^{\circ} \mathrm{C}$ colonies of microorganisms are present from both seeding samples (surface and deep), with higher prevalence (almost double) in samples taken from the surface of sheep carcasses with number colonies variation at 23 to $53(39,3 \pm 8,7)$ on the surface of the carcasses and 10-18 $(13,3 \pm 2,3)$ on the deep of the sheep carcasses. On samples taken from the carcasses of pigs (fig.2), the colonies of microorganisms predominated numerically in samples taken from the surface of the carcasses and only single colonies are grown from the samples from deep of carcasses. Meanwhile, samples taken from the carcasses of pigs, colonies of microorganisms had prevalent in samples taken from the surface of carcasses and unique colonies

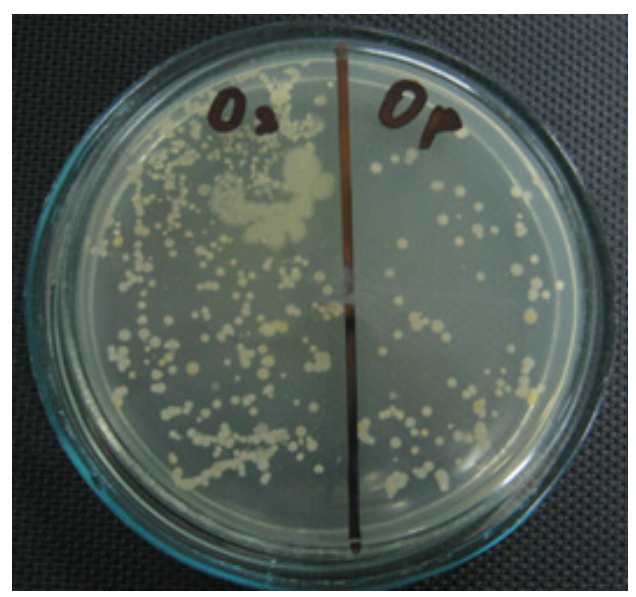

Fig. 1. Petri plates with peptone agar at 48 hours after incubation

(seeding from the sheep carcasses) 
are grown only from sowings which was made from samples from deep of pigs carcasses with variation from 12 to 24 of colonies $(16,0 \pm 3,0)$ on surface of the carcasses and 4-11 $(7,3 \pm 2,0)$ from the deep of the carcasses.

Morphological structure of colonies isolated from the carcasses of both animal species has the round or oval form, situated in piles oron form of chain specific for Streptococcus.

Also seeding was conducted on the nutrient medium Endo were samples were taken from the surface and deep of sheep and pigs carcasses. After 48 hours the colonies of microorganisms grown from both seeding samples (surface and deep of the carcasses). The variation of colonies

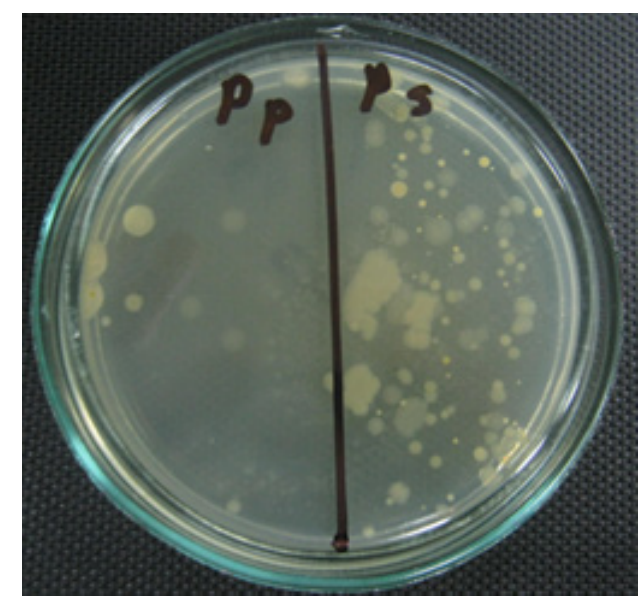

Fig.2. Petri plates with peptone agar at 48 hours after incubation

(seeding from the pigs carcasses)

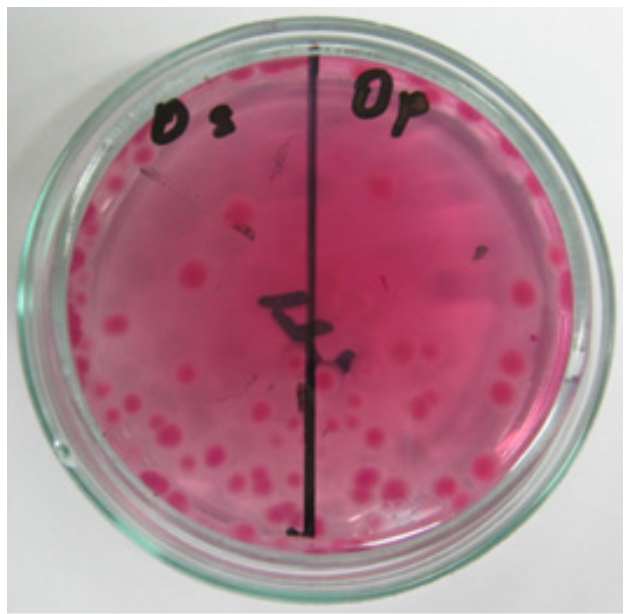

Fig.3. Petri plates with nutrient medium Endo at 48 hours after incubation (seeding from the sheep carcasses) number from the surface of sheep carcasses was at 20 to $31(24,6 \pm 3,2)$ in samples collected from the surface of the carcasses and at 3 to $9(8,6 \pm 3,1)$ in samples collected on deep of the carcasses.

The samples which were taken from the surface and deep of pig carcasses and seeding on nutrient medium Endo also demonstrated the presence of microbial flora predominated numerically in samples taken from the surface of the carcasses with red - metallic color and round or oval forms, placed separately on the surface of Petri plates which is characteristically for E. coli. The variation of number of colonies on the samples from the surface of the carcasses had variation between 7 to 12 colonies $(10,6 \pm 1,8)$ on surface of the carcasses and $6-11(8,6 \pm 1,4)$ on the deep of the carcasses.

Analyzing the obtained data is possible to mention that the largest number of microorganisms was developed from samples taken from the surface of the of sheep and pig carcasses especially with prevalent increased number on sheep carcasses. Compared to the samples collected from the deep of the carcasses the microorganism's colonies number was considerably smaller. The isolated colonies of microorganisms have characteristics of Streptococcus and E. coli microorganism's types. More multiple colonies of microorganisms have been established from the samples collected from the surface of sheep carcasses.

The growth of colonies of microorganism's in particular streptococcal microbial form was observed in the samples placed in the tubes with

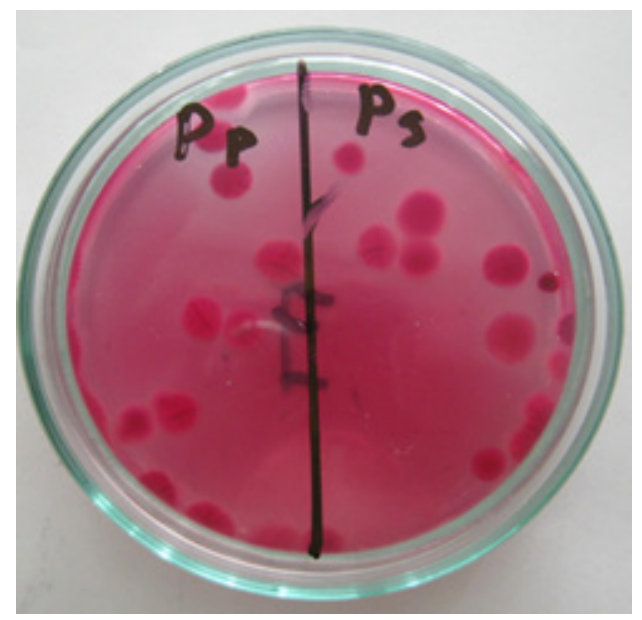

Fig. 4. Petri plates with nutrient medium Endo at 48 hours after incubation (seeding from the pigs carcasses) 


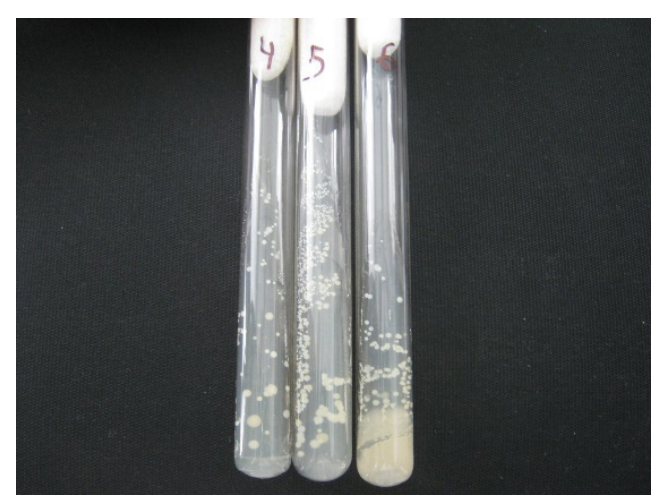

a)

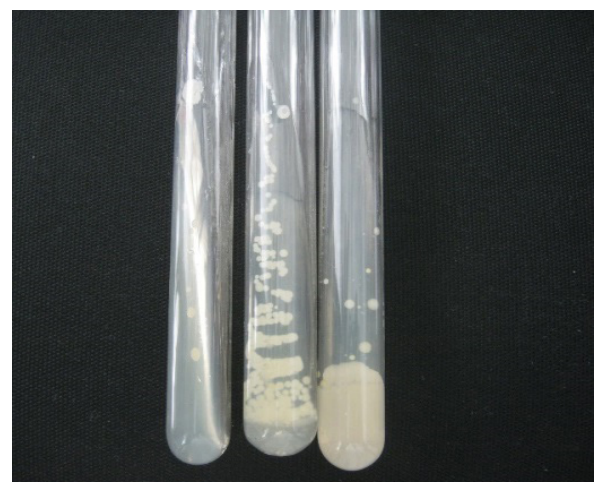

b)

Fig. $5(\mathbf{a}, \mathbf{b})$ The tubes with peptone broth medium at 48 hours after incubation (seeding from samples taken from the surface of the samples harvested from pig and sheep carcasses)

peptone agar (fig.6 a,b). The colonies has the same morphological structure, color and placement on the substrate surface as the as with seeding on Petri plates. More intensive growth of colonies of microorganisms was observed in environments were the samples was taken from the surface of sheep carcasses.

\section{CONCLUSION}

Bacteriological examination of samples taken from the carcasses of pig and sheep has shown the risk of contamination with bacterial micro flora, which is evident on the carcasses of sheep were the predominance types of microorganisms is $\mathrm{E}$. coli and Streptococcus.

Prevention of contamination of carcasses during the sale period can be monitored by maintaining of the temperature regime and to minimize contact with the environment vectors.

\section{REFERENCES}

1. Artur TM (2010). Super shedding of Escherichia coli 0157:H7 by cattle and the impact on beef carcass contamination. Meat Science 86:32-37.

2. Juneja VK, Melendres MV, Huang L, Subbiah J, Thippareddi $\mathrm{H}$ (2009). Mathematical modeling of growth of Salmonella in raw ground beef under isothermal conditions from 10 to $45^{\circ} \mathrm{C}$. International Journal of Food Microbiology 131:106-111.

3. Papadopoulou OS (2012). Transfer of foodborne pathogenic bacteria to non-inoculated beef fillets through meat mincing machine. Meat Science 90:865-869.

4. So Jung Kim, Dong Sun Lee. (2009). Simple microbial growth model applicable to dynamic temperature conditions: Evaluation of a nondimensional model. Journal of Food, Agriculture \& Environment 7(3\&4):192196.

5. Tian Ding, Rahman SME, Purev U, Deog-Hwan Oh (2010). Modelling of Escherichia coli 0157:H7 growth at various storage temperatures on beef treated with electrolyzed oxidizing water. Journal of Food Engineering 97:497-503. 